\title{
DERMATOFITIAS EN NIÑOS: REVISION RETROSPECTIY DE 487 CASOS EN TUCUMAN-ARGENTINA
}

\author{
(Dermatophytoses in children: Retrospective revision of 487 cases in \\ Tucumán-Argentina)
}

\begin{abstract}
Alvarez, C. ${ }^{1}$ Runco, R. ${ }^{1,2}$ y Salim, R. ${ }^{2}$
1.Laboratorio de Micología del Hospital del Niño Jesús. Pasaje Hungría 750 (4000) San Miguel deTucumán. R.Argentina. 2.Cátedra de Micología. Instituto de Microbiología «Dr. Luis C. Verna». Facultad de Bioquímica, Química y Farmacia - Universidad Nacional de Tucumán. Ayacucho 491 (4000) San Miguel deTucumán. R.Argentina. E-mail:bqco_chal@hotmail.com
\end{abstract}

Palabras clave: Revisión retrospectiva, dermatofitias, niños

Key words: Retrospective revision, dermatophytoses, children

\section{RESUMEN}

Los hongos dermatofitos son los agentes causales más frecuentes de micosis. No obstante, la prevalencia ą de los agentes causales varía en las regiones geográficas, las características demográficas de la población estudiada y la metodología de laboratorio. A fin de determinar la frecuencia de dermatofitos en niños de Tucumán (R. Argentina), se presentan los resultados de una revisión retrospectiva de 9 años (2000-2008)de los protocolos médicos de 712 niños de 0 a 14 años de edad, (413 varones y 299 mujeres), con diagnóstico clínico de micosis en piel, uñas, pelos y cuero cabelludo evaluados en el Hospital del Niño Jesús.

Las tineas fueron diagnosticadas en 487 pacientes $(68,4 \%)$, predominando en el género masculino. La distribución de los hongos aislados fue: M. canis $(78,4 \%)$, T. mentagrophytes $(6,4 \%)$, T. rubrum (6,2\%), T. tonsurans (4,5\%), M. gypseum (3,9\%), T. mentagrophytes var. interdigitale $(0,4 \%)$ yE. floccosum $(0,2 \%)$. Coincidentemente con trabajos previos realizados en nuestra provincia, la tinea capitis es la afección predominante y M. canis sigue siendo su principal agente patógeno más frecuente $(93,8 \%)$. La alta proporción de aislamientos de M. canis, indica claramente que las especies zoofilicas son las más prevalentes en nuestra área.

El grupo etario de mayor incidencia fue el de 0 a 3 años seguido del grupo de 4 a 6 años de edad.

Recibido el 2 de Septiembre 2009

Aceptado el 20 de Octubre 2009

\section{ABSTRACT}

Dermatophyte fungi are the most frequent agents causing mycosis. However prevalence of the causing agents varies with the geographic regions, demographic characteristics of the studied population and with the laboratory methodology. In or der to determine the incidence of Dermatophytes in chidren of Tucumán (R. Argentina), results of a 9-year retrospective revision (2000-2008) of the medical protocols of 712 children of 0 to 14 years of age (413 men and 299 women) diagnosed with mycosis in hair, scalp, skin and nails are reported and who had been evaluated in the Hospital del Niño Jesús. $T$ inea wer e diagnosed in 487 patients (68.4\%), prevailing in the masculine genus. The distribution of isolated fungi was: $\quad$ M.canis, (78.4\%), T.mentagrophytes (6.4\%), T.rubrum (6.2\%), T tonsurans (4.5\%), M.gypseum (3.9\%), T.mentagrophytes var interdigitale $(0.4 \%)$ and E.floccosum (0.2\%). Coincidently with previous studies accomplished in our province, tinea capitis is the prevailing affection whileM.canis continues being its main and most frequent pathogenic agent (93.8\%). The high number of M.canis isolations show clearly that zoophilic species are the most prevalent in our area. Children aged 0 to 3 year exhibited the highest prevalence followed by those of 4 to 6 year.

\section{INTRODUCCION}

Los dermatofitos son un grupo de hongos queratinofílicos, morfológica, fisiológica y taxonómicamente muy relacionados entre sí, capaces de invadir el 
estrato córneo y colonizar las estructuras queratinizadas (piel, pelo y uñas). Se encuentran entre los agentes fúngicos más comunes que infectan al hombre. $\mathrm{Su}$ frecuencia de presencia varía con la edad, el sexo, el origen étnico y los hábitos culturales y sociales de la población. Se estima que constituyen el tercer trastorno cutáneo más común en los niños menores de 12 años.

La dermatología pediátrica ha experimentado en los últimos años un desarrollo creciente y los pediatras conocen la frecuencia de los padecimientos cutáneos de sus pacientes, los cuales representan alrededor de la tercera parte de las consultas habituales, y en otros casos hasta el $40 \%$. En la infancia se pueden observar prácticamente todas las dermatosis de la edad adulta, sin embargo, ellas se presentan con diferentes frecuencias y, a veces, bajo diferentes formas clínicas (Caballero, 2004).

El aumento de este tipo de patología en las últimas décadas se debe tanto al incremento de los viajes y desplazamientos de la población mundial como a la aparición de nuevos fármacos inmunosupresores utilizados en tratamientos de otras enfermedades que conllevan a modificaciones en la clínica. En los niños el problema aumenta dada la facilidad de contagio que existe en la intensa relación que habitualmente se mantiene en la edad escolar y en los jardines de la primera infancia (Ruíz, 2004). Es difícil determinar su prevalencia, debido a que esas infecciones no se consideran de denuncia obligatoria. Las revisiones fragmentarias de estudios epidemiológicos y comunicaciones de casos indican que estas micosis son unas de las enfermedades humanas más comunes, con alto grado de morbilidad y pueden ser las más prevalentes de todas las infecciones humanas (García Díaz, 1995; Pérez Cárdenas, 2005).

La tinea capitis es una infección del cuero cabelludo, producida por varias especies de dermatofitos, los cuales causan la mayoría de las micosis superficiales. Existen dos formas, las tineas microspóricas o bien, las tineas tricofíticas, cuyas placas alopécicas no tienen límites tan netos como las microspóricas. Los pacientes más frecuentemente afectados por tinea capitis son niños entre 4 y 14 años, siendo rara en adultos. Es un trastorno de la población pediátrica hasta la pubertad, época en la que deja de constituir una infección importante. Su frecuencia es mayor en la niñez, principalmente entre los 5 y 10 años de edad; a partir de los 11 años, se observa un descenso pronunciado. También se han descrito casos en menores de 1 año y en adultos en edad avanzada, sobre todo en mujeres post-menopáusicas (Brito, 2001; Ruíz, 2004).

La tinea corporis (o tinea de la piel lampiña) es una enfermedad cosmopolita que afecta a las personas de cualquier edad y sexo y es más frecuente en niñosEn este su localización tiene preferencia por la cara. Tal como lo demuestra la literatura especializadsu frecuencia es menor que la tinea capitis y está muy asociada al contacto con animales domésticos (Ruíz, 2004).

La tinea unguium, también conocida como onicomicosis dermatofítica, es la forma clínica menos común en niños, pero con un incremento progresivo en su frecuencia. EnAmérica Latina no es tan rara (4-8\%). Se cree que factores climáticos y socioeconómicos pueden jugar un papel importante. La menor frecuencia en niños se debería a un contacto reducido con el hongo, menor tiempo de exposición a ambientes con alta densidad de hifas y esporas infectantes, rápido crecimiento ungueal comparado con la población adulta, menor área de superficie ungueal disponible, menor exposición a traumas que faciliten la colonización, diferencias estructurales en las uñas de los pies y una menor prevalencia de tinea pedís en la edad pediátrica (Vllanueva Reyes, 2006).

En los niños la onicomicosis dermatofítica afecta principalmente las uñas de los pies. El diagnóstico se realiza por la sospecha clínica, siendo necesario tener presente que las onicomicosis representan un porcentaje importante de las onicodistrofias pero no la totalidad, motivo por el cual, no debe obviarse nunca el estudio micológico antes de establecer el tratamiento antifúngico. Por ende, la búsqueda intencionada para medir la frecuencia real y realizar una correlación, tanto en el ámbito clínico, micológico e histopatológico, permite un conocimiento más adecuado acerca de la etiopatogenia y la historia natural de la onicomicosis en niños; al mismo tiempo permite desarrollar mejores estrategias en el estudio, prevención y tratamiento, que evitan la distrofia ungueal temprana y la diseminación de la infección en este grupo de pacientes (Runco, 2000; Villanueva Reyes, 2006).

El propósito de este estudio, es establecer la incidencia de las diversas especies de dermatofitos en nuestro medio en niños de 0 a 14 años de edad, con diagnóstico presuntivo de micosis en cuero cabelludo, piel y uñas.

\section{MATERIALES YMETODOS}

Para evaluar frecuencia de los agentes causales de micosis que afectan la piel, pelos y uñas, se realizó una revisión retrospectiva de 9 años de los protocolos médicos de 712 niños, internados y/o ambulatorios, de 0 a 14 años de edad, (413 varones $(58,0 \%)$ y 299 mujeres $(41,9 \%$ фon sospecha clínica de micosis que fueron evaluados tanto en la consulta del Servicio de Dermatología como de otros servicios del Hospital del Niño Jesús de Tucumán durante los años 2000 al 2008. Se recopilaron los siguientes datos: edad, sexo, localización anatómica y resultados de los estudios micológicos (examen directo previa aclaración con $\mathrm{KOH}$ y cultivo). Tanto el procesamiento de las 
muestras como la identificación del agente causal se realizaron en el Laboratorio de Micología del mismo Hospital.

Según el tipo de lesión las muestras se tomaron por raspado con bisturí estéril o extracción con pinza de depilar, en pacientes previamente preparados según las normas establecidas para estos casos. No se tomaron en cuenta aquellos protocolos médicos en los que no se realizó examen micológico directo y cultivo, o que consignen que el paciente hubiese recibido previamente terapia antimicótica tópica.

Todas las muestras fueron recogidas entre dos portaobjetos esterilizados por flameado. Parte de ellas se observaron al microscopio entre portaobjetos y cubreobjetos, previa aclaración con hidróxido de potasio al 20$30 \%$ en caliente, y el resto se sembró en medio de Sabouraud-Glucosa-Agar adicionado comtibiót i c(Psenicilina $50 \mathrm{UI} / \mathrm{ml}$ - Estreptomicina $\$ \mathrm{~g} / \mathrm{ml}$ ). Todos los tubos fueron incubados a $28^{\circ} \mathrm{C}$ durante un lapso de 15 a 21 días, observados semanalmente en busca de desarrollo fúngico, y pasadas las 3 semanas fueron informados como negativos ante la falta de desarrollo.

La identificación de los dermatofitos se realizó siguiendo los criterios de Rebell yTaplin (1979), Kane et al., (1997); De Hoog et al., (2000), incluyendo: estudios macro y micromorfológicos de los cultivos, hidrólisis de urea y producción de órganos perforadores del pelo in vitro.

La población infantil estudiada comprende a pacientes de un estrato social especial que solamente puede tener acceso a la consulta médico-asistencial gratuita hospitalaria.

Metodología estadística: se trata de un estudio descriptivo, retrospectivo, donde se utilizaron valores absolutos y porcentajes.

\section{RESULTADOS}

Del total de muestras procesadas (712), se diagnosticaron dermatofitias en 487 pacientes $(68,4 \%)$. La localización más frecuente fue tiña capitis $(76,6 \%)$, seguida de tiña corporis $(17,0 \%)$, y en último orden de frecuencia se encontraron 31 lesiones ungueales $(6,4 \%)$. El porcentaje de resultados negativos totales obtenidos en los cultivos fue del 31,6\% (Tbla.1).

La mayor prevalencia la presentaron los individuos del sexo masculino $(56,0 \%)$ y un $(44,0 \%)$ al género femenino.

La tiña capitis es la afección predominante y $\boldsymbol{M}$. canis $(93,8 \%)$ sigue siendo su principal agente patógeno seguido de T. tonsurans (3,5\%) yM. gypseum (1,9\%).T. mentagrophytes $(0,8 \%)$ fue el agente menos frecuente (Fig. 1). Respecto a la distribución de los agentes que cau-
Tabla 1. Distribución de las micosis por agentes causales

\begin{tabular}{|c|c|c|c|}
\hline MICOSIS & $\begin{array}{c}\text { AGENTE } \\
\text { ETIOLOGICO }\end{array}$ & $\begin{array}{c}\mathrm{N}^{\circ} \\
\text { casos }\end{array}$ & $\%$ \\
\hline \multirow{4}{*}{$\begin{array}{c}\text { TIÑA } \\
\text { CAPITIS } \\
(373) 76,6 \%\end{array}$} & M. canis & 350 & 93,8 \\
\hline & T.tonsurans & 133,5 & \\
\hline & M. gypseum & 71,9 & \\
\hline & T. mentagrophytes & 30,8 & \\
\hline \multirow{7}{*}{$\begin{array}{c}\text { TIÑA } \\
\text { CORPORIS } \\
\text { (83) } 17 \%\end{array}$} & M. canis & 32 & 38,5 \\
\hline & T. mentagrophytes & 1923 & 0 \\
\hline & M. gypseum & 1214 & 5 \\
\hline & T. tonsurans & 910 & 8 \\
\hline & T. rubrum & 910 & 8 \\
\hline & $\begin{array}{l}\text { T. mentagrophytes var. } \\
\text { interdigitale }\end{array}$ & 11,2 & \\
\hline & E. floccosum & 11,2 & \\
\hline \multirow{3}{*}{$\begin{array}{c}\text { TIÑA } \\
\text { UNGUIUM } \\
(31)(6,40 \%)\end{array}$} & T. rubrum & 21 & 67,8 \\
\hline & T. mentagrophytes & 929 & 0 \\
\hline & $\begin{array}{l}\text { T. mentagrophytes var. } \\
\text { interdigitale }\end{array}$ & 13,2 & \\
\hline TOTAL & & 487 & 100,0 \\
\hline
\end{tabular}

san lesiones en piel, una vez más $\boldsymbol{M}$. canis $(38,5 \%)$ es el que presenta la mayor frecuencia seguido de $\boldsymbol{T}$. mentagrophytes (23\%), M. gypseum (14,5\%), T. tonsurans $(10,8 \%)$, T. rubrum $(10,8 \%)$ T. mentagrophytes var. interdigitale (1,2\%) yE. floccosum (1,2\%) (Fig.2).

Tanto en las muestras de pelos y cuero cabelludo como en las de piel se encontró un franco predominio de M. canis $(78,4 \%)$ responsable del $93,8 \%$ de tinea capitis y del $38,5 \%$ de tinea corporis (Fig. 1 y 2).

T. rubrum es el dermatofito más frecuente en lesiones en uñas $(67,8 \%)$, seguido de $\boldsymbol{T}$. mentagrophytes (29\%) y T. mentagrophytes var. interdigitalis (3,2\%) (Fig. 3).

El grupo etario de mayor incidencia fue el de 0 a 3 años seguido del grupo de 4 a 6 años de edad.

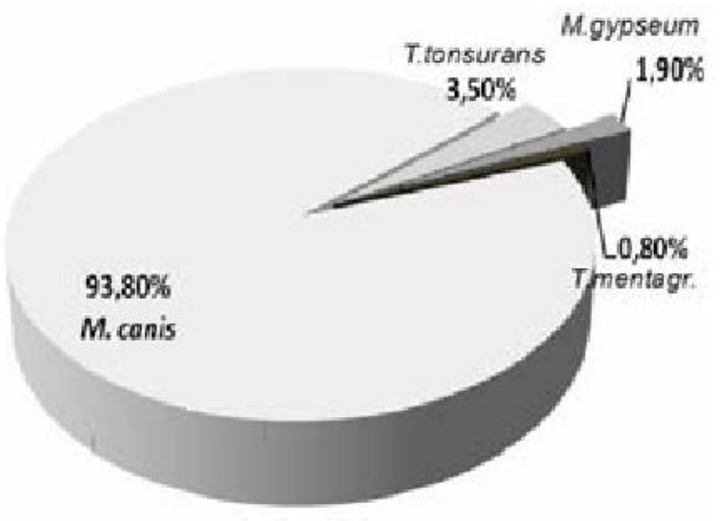

Fig. 1. Frecuencia de agentes causantes de tinea capitis 


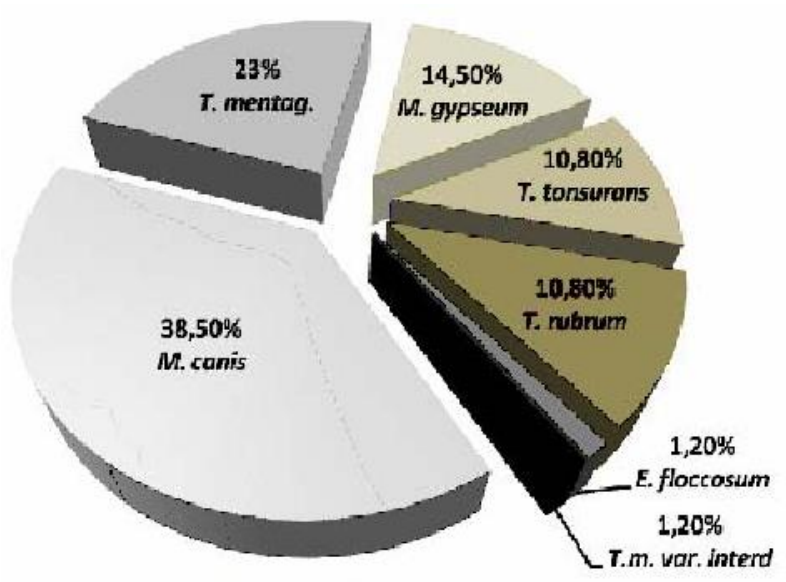

Fig.2. Distribución de agentes etiológicos de tinea corporis

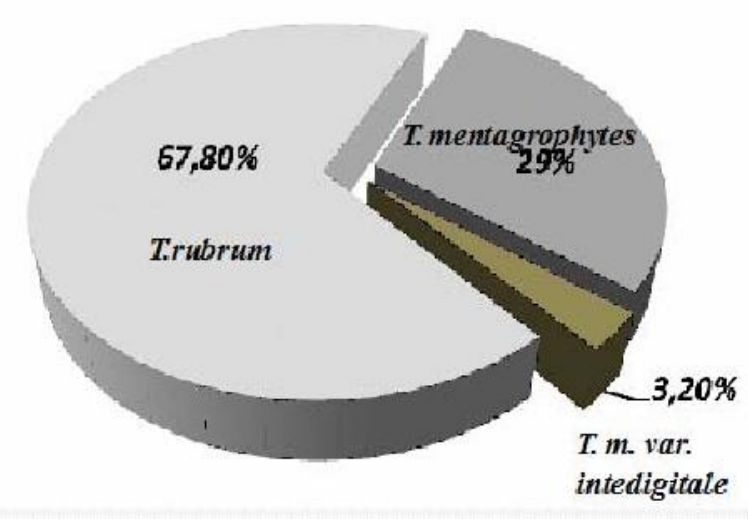

Fig.3. Distribución de agentes etiológicos de tinea unguium

\section{DISCUSION}

Las dermatofitias se encuentran en el séptimo lugar de las dermatosis más frecuentes en la infancia y en general son parte importante del motivo de consulta en la práctica diaria del dermatólogo, siendo las micosis superficiales las más frecuentes, hecho que también se refleja en nuestro estudio. Dentro de éstas, las dermatofitias en cabeza y cuerpo ocupan la mayor proporción, con una incidencia mayor en la edad escolar o antes de la pubertad, mientras en tinea pedis y unguium son menos frecuentes (Bianchi, 1988; Runco, 2000; Brito, 2001; Caballero, 2004; Pérez Cárdenas, 2005).

Si bien las afecciones provocadas por los dermatofitos son frecuentes, superficiales y benignas en la mayoría de las personas, su epidemiología experimenta modificaciones regulares, especialmente en el caso de las tineas, El desempeño del laboratorios es esencial debido a que las técnicas de toma de material evolucionan, pero también lo hacen los medios de identificación de las especies involucradas. Sin embargo, el reconocimiento de las lesiones provocadas por estos hongos, así como los aspectos inherentes a su fisiopatología, epidemiología, evolución e inclusive tratamiento, son de importancia a la hora de procesar una muestra. En los últimos quince años, el arsenal terapéutico se ha enriquecido gracias a nuevos antimicóticos más adecuadas para los diferentes sitios afectados y por nuevas moléculas (tratamientos locales y generales) (Pérez Cárdenas, 2005)

Los datos obtenidos muestran escasas diferencias con aquellos producidos por estudios similares (Bianchi, 1988; Davel, 1999; Ruíz, 2004; Pérez Cárdenas, 2005; Villanueva Reyes, 2006) y con trabajos previos realizados en nuestra provincia (Elías, 1978; Elías, 1981; Runco, 2000): la tinea capitis es la infección fúngica más frecuente con predominio en el sexo masculino y en el grupo etario de preescolares y escolares, siendo M. canis el agente causal más aislado. En el presente estudio esta especie alcanzó cifras casi idénticas $(79,3 \%)$ a las de Runco et al., (2000) en piel, pelos y cuero cabelludo, sin embargo,

M.gypseum y $T$ rubrum se presentaron en menor cantidad, mientras T.mentagrophytes mantuvo porcentajes similares. No encontrándose otras especies ni variedades distintas de las mencionadas.

El alto porcentaje de aislamientos de M. canis, a diferencia con M. gypseum, indica que las especies zoofílicas son las más prevalentes en nuestra área.

Coincidiendo nuevamente con la literatura antes mencionada, el segundo lugar en el orden de frecuencia lo ocupó la tinea corporis, yM. canis representó la especie más frecuente, seguida por

T. ment agrophytes, $M$. gypseum y T.tonsurans.

Al igual que lo descrito en otros estudios, el agente causal de tinea unguium aislado con mayor frecuencia fue T. rubrum (Hernández-Salazar, 2007). M. canis sigue siendo el dermatofito más frecuente en tinea capitis y T. ment agrophytes el más común en otras localizaciones.

Las diferentes lesiones producidas por dermatofitos se han asociado con grupos poblacionales, sus condiciones socioeconómicas y la época en que se hicieron los estudios. Sin embargo, no hay consenso en cuanto a cual es la lesión más frecuente, asimismo, no siempre existe un predominio de una especie de dermatofito en particular. No obstante, observamos que en nuestra provincia los resultados son similares en el tiempo.

En conclusión, podemos sostener que las micosis más frecuentes en la población pediátrica de Tucumán son las dermatofitias, y entre ellas, la tinea capitisseguida de la tinea corporis. 


\section{REFERENCIAS}

Bianchi, M.; Robles, A.M. \& Arechavala A. I. (1988). Estadística de las especies causales de Dermatofitias en el Hospital de Infecciosas «Francisco Javier Muñiz». Rev . Arg. Micología 1 1:24-6

Brito, A.; Mar cano, C.; Rivas, G .; Rodríguez, F . (2001). Dermatofitos causantes de Tiña capitis en niños y adolescentes. Rev. Soc. Ven. Microbiol . 21:26-28

Caballer o, G .; Knopfelmacher , O. \& Bolla de Lezcano, L. (2004). Dermatosis de consulta más frecuente en dermatología pediátrica. Pediatría 31:7-16

Davel, G .; Perr otta, D.; Canter os, C.; Córdoba, S.; Roder L.; Brudny, M.; Abrantes, R. (1999). Multicenter study of super ficial mycoses in Argentina. Rev . Argent. Microbiol 31:173-81

García Díaz, R.; González, M.T ; Santos, P .\& Pierini, A.M. (1995). Superficial mycoses in children: a 3-year survey in Buenos Aires. En: Pierini AM, García-Díaz R, Bustamante R.E. Editores. Pediatric Dermatology . Buenos Aires: Elsevier . pp. 75-78

Hernández-Salazar , A.; Carbajal-Pruneda, P .; Fernández Mar tínez, R.; Arenas, R. (2007). Dermatofitosis por Trichophyton rubrum . Experiencia de 10 años (1996-2005) en un servicio de dermatología de un hospital general de la Ciudad de México. Rev. Iberoam. Micol. 24: 122-124

Hoog, G.S. de.; Guarr o, J.; Gené, J. \& Figueras, M.J. Atlas of Clinical fungi. 2nd ed. CBS-Univ . Rovira i Virgili
Kane, J.; Summerbell, R.; Sigler ～， L.; Krajden, S.; Lands, G • (1997). Laboratory Handbook of Dermatophytes. A clinical guide and laboratory manual of dermatophytes and other filamentous fungi from skin, hair , and nails. S tar Publishing Company, Belmont, CA

Pér ez Cárdenas, J. E. (2005). Aspectos actuales sobre las dermatofitosis y sus agentes etiológicos. Biosalud, 14:10-121

Rebell, G . \& Taplin D. (1979). Dernatophytes: their recognition and identification 3d ed., Univ . Miami Press, Miami

Ruiz, A.; Cir oco, A.; González, F.; Sáenz, A.; Ferr eir o, M. (2004). Micosis cutáneas en la Infancia: estudio retrospectivo desde 1990 hasta el 2001 en la consulta de micología del Servicio de Dermatología del Hospital Universitario de Caracas.

Dermatología V enezolana. 42:30-34

Runco de Laborda, R.; Salim, R. \& Silva, J. Dermatomicosis en niños y diagnóstico presuntivo: cinco años de estudios en Tucumán- Argentina. Boletín Micológico 15:57-63

Villanueva-Reyes, J. \& Arenas, R. (2006). Onicomicosis en niños: estudio en una población mexicana. Dermatol. Pediatr . Lat 4:197-203 